\title{
Colangiografía endoscópica en anatomía modificada: ¿Percutánea, enteroscopia, USE, EDGE, cirugía?
}

\author{
Félix Ignacio Téllez-Ávila* \\ Departamento de Endoscopia Gastrointestinal, Instituto Nacional de Ciencias Médicas y Nutrición Salvador Zubirán, Ciudad de México, México
}

\begin{abstract}
Resumen
De manera uniforme, la colangiopancreatografía retrógrada endoscópica (CPRE) se considera como uno de los procedimientos con mayor grado de dificultad en la endoscopia gastrointestinal. Ello debido a la cantidad de maniobras simultáneas que se requieren para ingresar a la vía biliar o al conducto pancreático. En pacientes que han sufrido modificaciones quirúrgicas de su anatomía el grado de complejidad aumenta. En algunas de estas cirugías han resecado o excluido algún segmento anatómico que hace imposible el acceso a la papila mayor o a la anastomosis bilioentérica de manera tradicional. Cualquiera que sea el caso, antes de iniciar el procedimiento de CPRE en los pacientes con anatomía modificada quirúrgicamente es indispensable que el endoscopista entienda muy bien qué tipo de cirugía tiene el paciente y que cambios son los que va a encontrar. Con lo anterior el médico podrá planear que equipo en específico necesita, cuáles son los accesorios con los que debe contar, que posición debe colocar al paciente y el tipo de anestesia que solicitará.
\end{abstract}

Palabras clave: Cinematografiaría retrógrada endoscópica (CPRE). Endoscopia gastrointestinal. Anastomosis bilioentérica.

Actualmente existen 4 opciones para acceder a la vía biliar de os pacientes que requieren intervenciones biliopancreáticas y que tienen anatomía modificada: vía percutánea, por enteroscopia, guiada/asistida por USE (incluyendo CPRE asistida por ultrasonido endoscópico, EDGE por sus siglas en inglés) o cirugía. El objetivo del presente trabajo es presentar los trabajos, a juicio del autor, más relevantes presentados en la semana de enfermedades digestivas (DDW) 2019 celebrada en San Diego.

Sin lugar a dudas la cirugía y el acceso percutáneo son métodos probados desde hace años y cada uno ha dejado claro su utilidad en los pacientes, sin embargo, también ha quedado claro que no son métodos ideales y que la tasa de complicaciones, tiempo de hospitalización y costos han llevado a los médicos involucrados a buscar mejores opciones. El uso de enteroscopia asistida con sobretubo se ha posicionado en los años recientes como el método de elección para intervenir la vía biliar en los pacientes con anatomía modificada ya que tiene una buena tasa de éxito clínico y las complicaciones son menores que los métodos inicialmente mencionados. Como prueba de lo anterior Krishnamoorthi, et al. (Sa1469) ${ }^{1}$ presentaron una revisión sistemática y meta-análisis en donde se evaluaron los resultados de las CPRE asistida por enteroscopia con sobretubo (CPRE-AES) incluyendo equipos monobalón, doble balón y espiral. La tasa de éxito para llegar a la papila fue de $80.1 \%$ (IC95\%: 73.6-83.5) y el éxito clínico (canulación de la papila y realizar tratamiento necesario) del 68\% (IC95\%: 64-71.6). En un meta-análisis presentado por Ayoub, et al. (Tu2038) ${ }^{2}$ se compararon los

\section{Correspondencia:}


resultados de la CPRE asistida por enteroscopia (CPRE-EC) vs la CPRE asistida por laparoscopia (CPRE-Lap). Se incluyeron 14 estudios de CPRE-Lap y 12 de CPRE-EC. El éxito clínico fue mayor para la CPRE-Lap (97.8\% vs $73.2 \%$ ) sin embargo el tiempo del procedimiento $(158 \pm 20$ vs $100 \pm 19 \mathrm{~min})$ y la tasa de complicaciones (19\% vs $6.5 \%$ ) también lo fueron. Por lo cual decidir que método utilizar en un paciente determinado debe considerar dichos factores.

Como se puede ver en los datos presentados previamente, claramente existe necesidad de nuevas opciones para el abordaje de estos pacientes. Runge, et al. $(1026)^{3}$ presentaron en un estudio multicéntrico sus resultados en relación con la CPRE asistida por USE (EDGE), se evaluaron los eventos adversos a largo plazo de pacientes sometidos a EDGE con especial atención a la persistencia de la fístula gastrogástrica (FGG). En dicho estudio se incluyeron los resultados de 12 centros en 5 años en donde se evaluaron 166 pacientes en total (2.7 pacientes/centro/año). El $51 \%$ de los casos se realizó en una sola sesión. El éxito técnico se alcanzó en 98\% (163/166) con un tiempo promedio de 87 minutos. En relación a la FGG, el cierre inicial se realizó con sutura en $31 \%$, con clips en $4 \%$, OVESCO en $9 \%$, en $28 \%$ de los pacientes se realizó solo "revivir" bordes con argón y en $27 \%$ de los casos no se realizó ninguna maniobra para promover el cierre. Se reportaron las complicaciones intraprocedimiento $(n=11 ; 6.6 \%)$ y a largo plazo $(n=17 ; 10.2 \%)$. Las más frecuentes fueron la perforación $(n=7)$ y persistencia de la $F G G(n=10)$, respectivamente. A 85 pacientes se les realizó algún estudio para búsqueda de persistencia de la FGG después del retiro de la LAMS y se corroboró en $10(12 \%)$. De estos 10 , en 7 se intentó cierre endoscópico el cual fue exitoso en $5 / 7$ (71\%). La conclusión fue que la EDGE es segura a corto y largo plazo.

Otro estudio retrospectivo de un solo centro en donde solo utilizaron prótesis de $20 \mathrm{~mm}$ de diámetro
(Su1167) ${ }^{4}$ se reportaron resultados de 9 pacientes vistos en 9 meses con éxito técnico y clínico de 100\% y la mayoría de los procedimientos (7/9) se realizaron en dos sesiones en un tiempo promedio de 32 minutos para la primera sesión y 44 minutos para la segunda. No se reportaron eventos adversos.

En otro estudio realizado en pacientes con cambios post-quirúrgicos diferentes a la $Y$ de Roux (Tu1983) ${ }^{5}$ se evaluó la seguridad y eficacia de utilizar LAMS para crear entero-entero anastomosis para realizar CPRE. Fue un estudio multicéntrico y retrospectivo de la experiencia de 8 centros en 5 años. Se incluyeron 18 pacientes en total (0.4 pacientes/año/centro). El éxito técnico fue de $94 \%$ (17/18) con tiempo promedio de 73 minutos con éxito clínico en los 17 pacientes en un seguimiento a 88 (54-142) días. Se reportaron 2 pacientes con eventos adversos.

\section{Conflicto de intereses}

No existen conflictos de interés. El autor recibió una beca completa para acudir al congreso DDW 2019 por parte de la Asociación Mexicana de Endoscopia Gastrointestinal.

\section{Bibliografía}

1. Krishnamoorthi R, Jayaraj M, Priyan $\mathrm{H}$, et al ERCP with overtube-assisted enteroscopy in patients with Roux-en-Y gastric bypass anatomy: a systematic review and meta-analysis. DDW; 2019 mayo 17-21; San Diego, CA. Sa1469.

2. Ayoub F, Brar T, Banerjee D, Abbas A, Yang D, Draganov P. Laparoscopy-assisted versus enteroscopy-assisted ERCP in patients with Rouxen-Y gastric bypass: a meta-analisys. DDW; 2019 mayo 17-21; San Diego, CA. Tu2038.

3. Runge T, Kowalski T, Baron T, et al. Living on the edge - success, longterm complications, and implications following EUS-directed transgastric ERCP: a multicenter study. DDW; 2019 mayo 17-21; San Diego, CA. 1026.

4. Hsueh W, Krafft M, Abdelqader A, Nasr J. EUS-directed transgastric ERCP with $20 \mathrm{~mm}$ lumen-apposing metal stents in patients with Rouxen-Y gastric bypass, are we closer to perfection. DDW; 2019 mayo 17-21; San Diego, CA. Su1167.

5. Yang J, James T, Baron T, et al. EUS-guided creation of entero-enterostomy using lumen apposing metal stents for pancreaticobiliary access in non-RYGB surgical anatomy patients. DDW; 2019 mayo 17-21; San Diego, CA. Tu1983. 